\title{
Psychological Defence Mechanisms in Patients on Chronic Haemodialysis Program
}

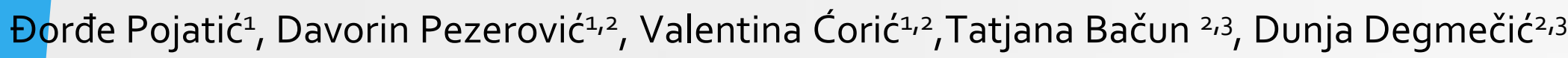

${ }^{1}$ General County Hospital Vinkovci

${ }^{2}$ Faculty of Medicine Osijek, Josip Juraj Strossmayer University of Osijek, Osijek, Croatia

3Clinical Hospital Centre, Osijek, Croatia

Background:Depending on the structure of their ego, an individual may use neurotic, immature, or mature defence mechanisms.Defence mechanisms have hyerchical order according to level in which they distort reality in order to prevent frustrations. Mature defenses are most adaptable, while immature defenses have high detrimental effect on psychological functioning(1). So far we know that type and levels of defenses have important contribution on severity index of inflammatory bowel diseases (2). It is uknown if defensive profile of patient can be important in predicting a quality of haemodialysis through biochemical findings.

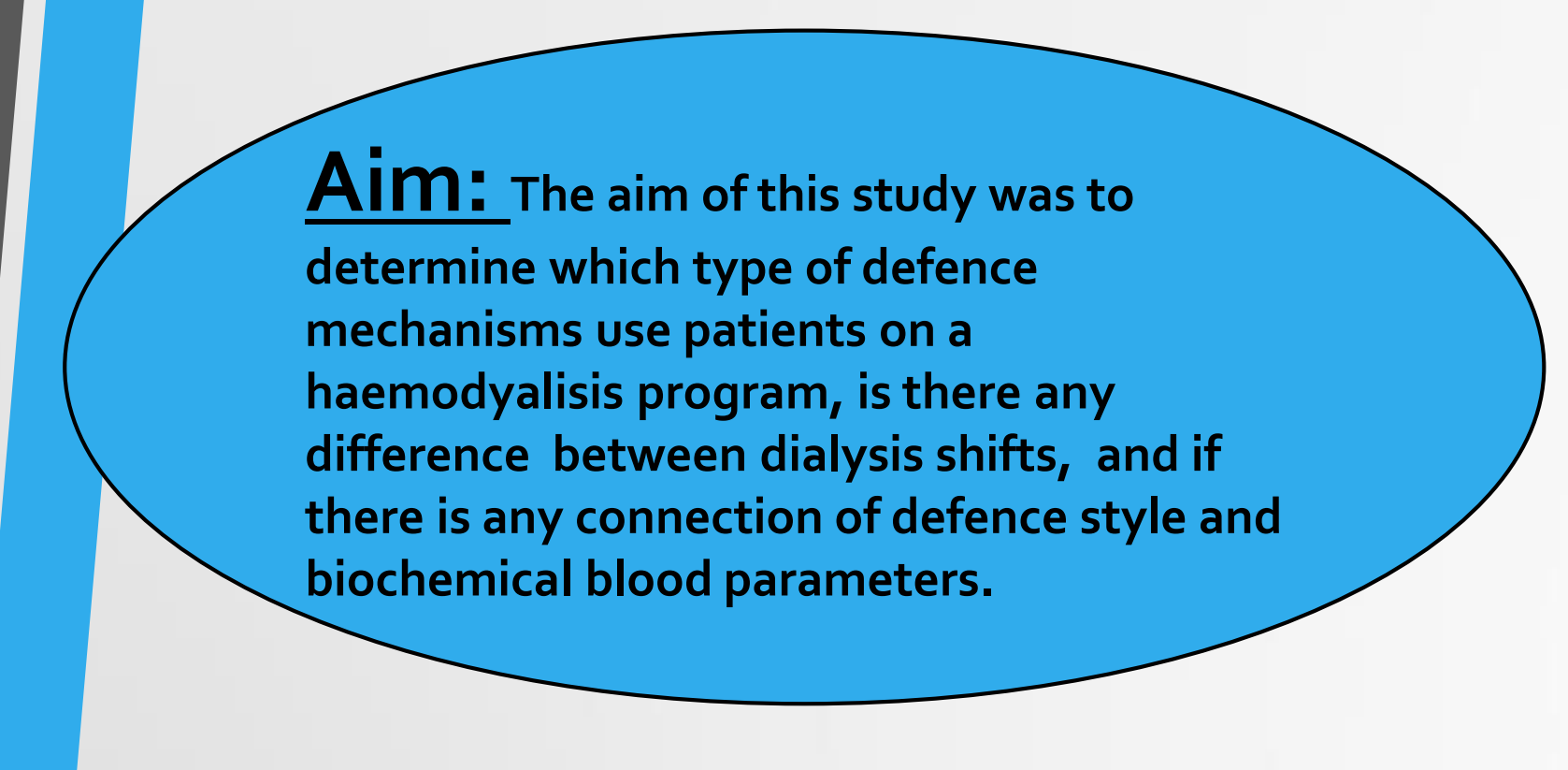

Aim: The aim of this study was to determine which type of defence chisms use patients on a difference between dialysis shifts, and if there is any connection of defence style and biochemical blood parameters.
Participants and methods: survey included 76 patients on chronic haemodialysis program in three shifts, 42 men (mean age $63,2 \pm 13,95$ years) and 36 women (mean age $64,7 \pm 13,53$ years), which were on chronic haemodialysis program longer than 3 months. We collected data using sociodemographic questionnaire and the self-report scale Defence Style Questionnaire-40 (DSQ-40), which was previously validated in sample of Croatian population (3). As part of laboratory tests for routine check-up of patients on the haemodialysis treatment program, we measured the serum levels of urea, creatinine, electrolytes, parathyroid hormone (PTH) and uric acid before the heamodialysis.

\section{Results:}

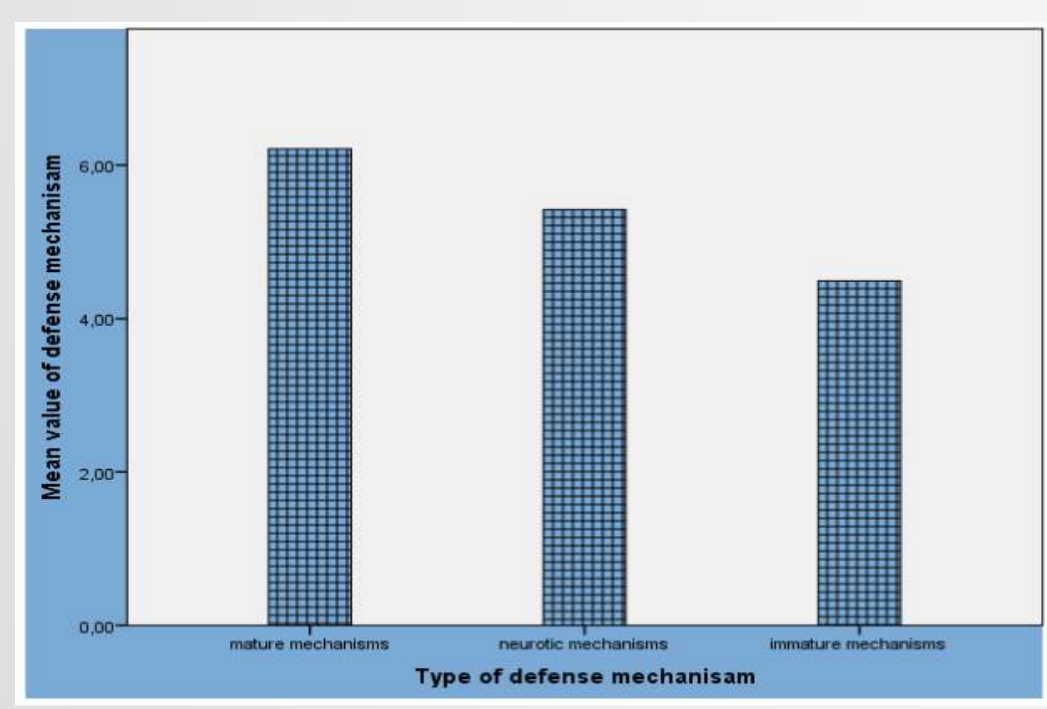

Patients use higher levels of mature defence mechanisms compared to immature mechanisms (One-Way ANOVA, $p=0.000$ ) and neurotic defence mechanisms compared to immature mechanisms (One-Way ANOVA, $p<0,01$ ).

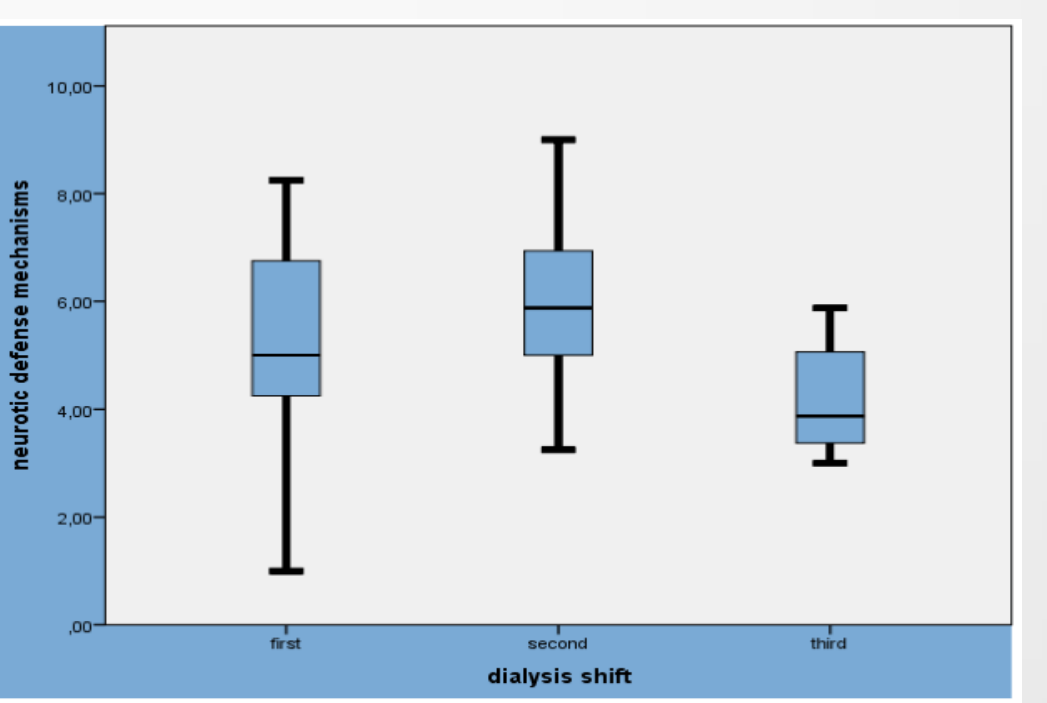

Patients in third shift use significantly higher levels of neurotic defenses compared to other two shifts (One-Way ANOVA, $p=0,020$ ).

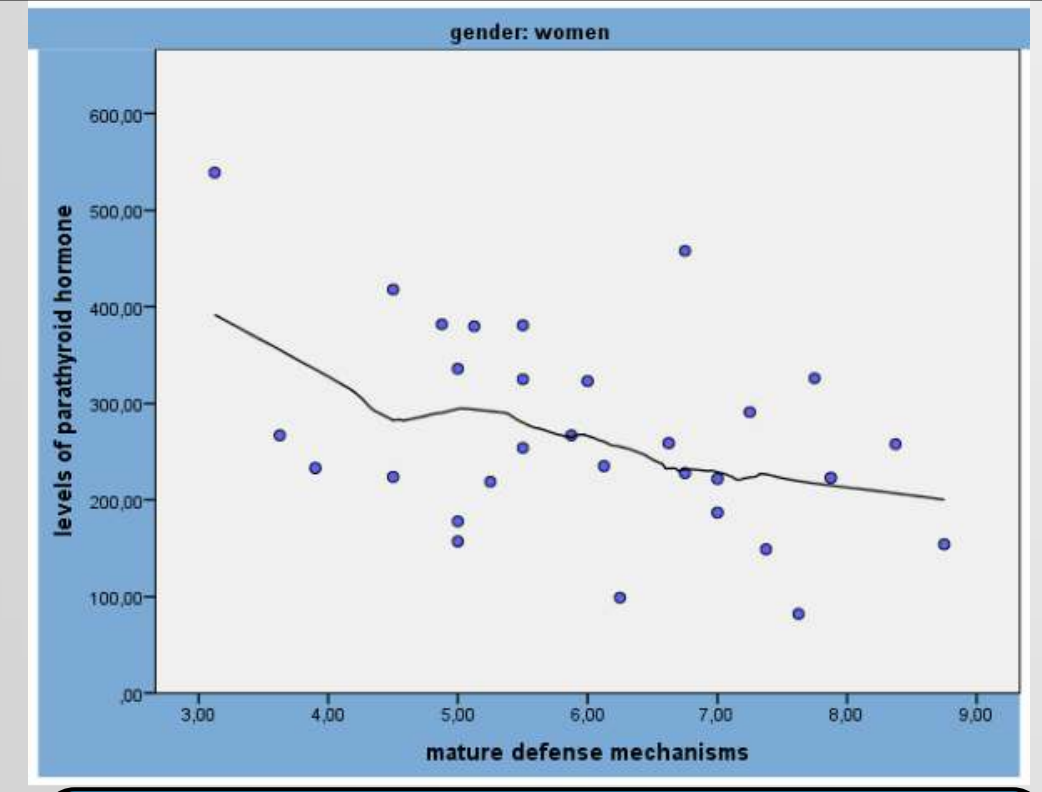

Mature defenses are in negative correlation with levels of PTH in females (Pearson coef. $=-, 423, p=, 020$ ).

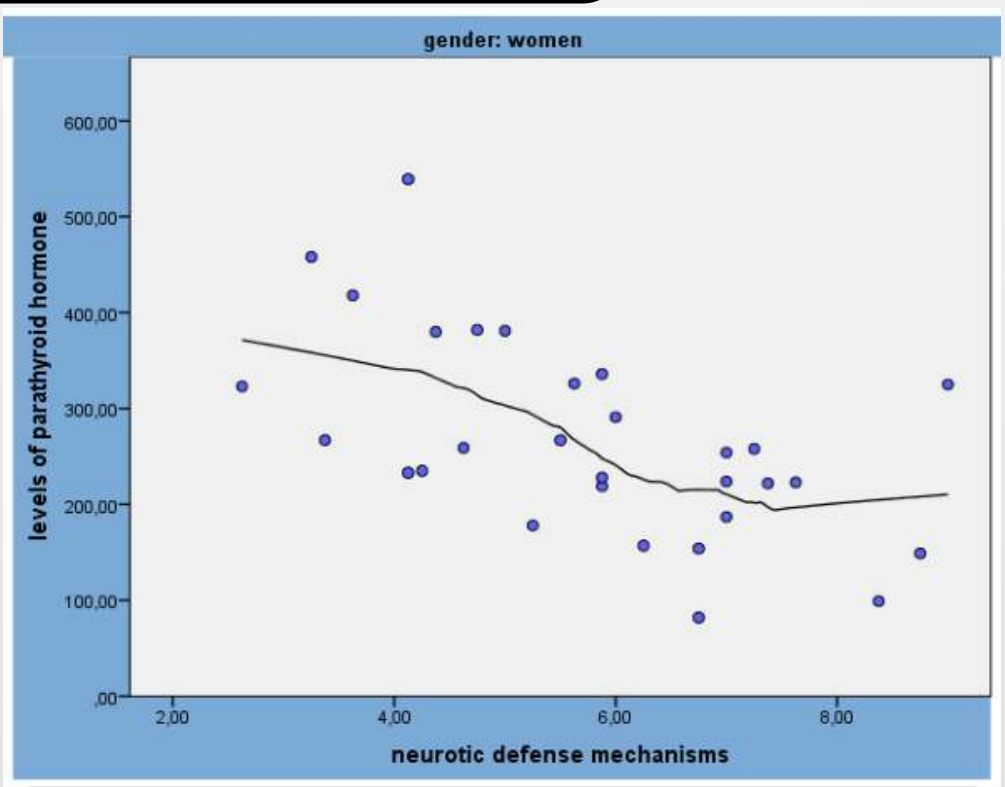

Neurotic defenses are in negative correlation with level of PTH in females (Pearson coef. $=-, 579, \mathrm{p}<0,001$ )

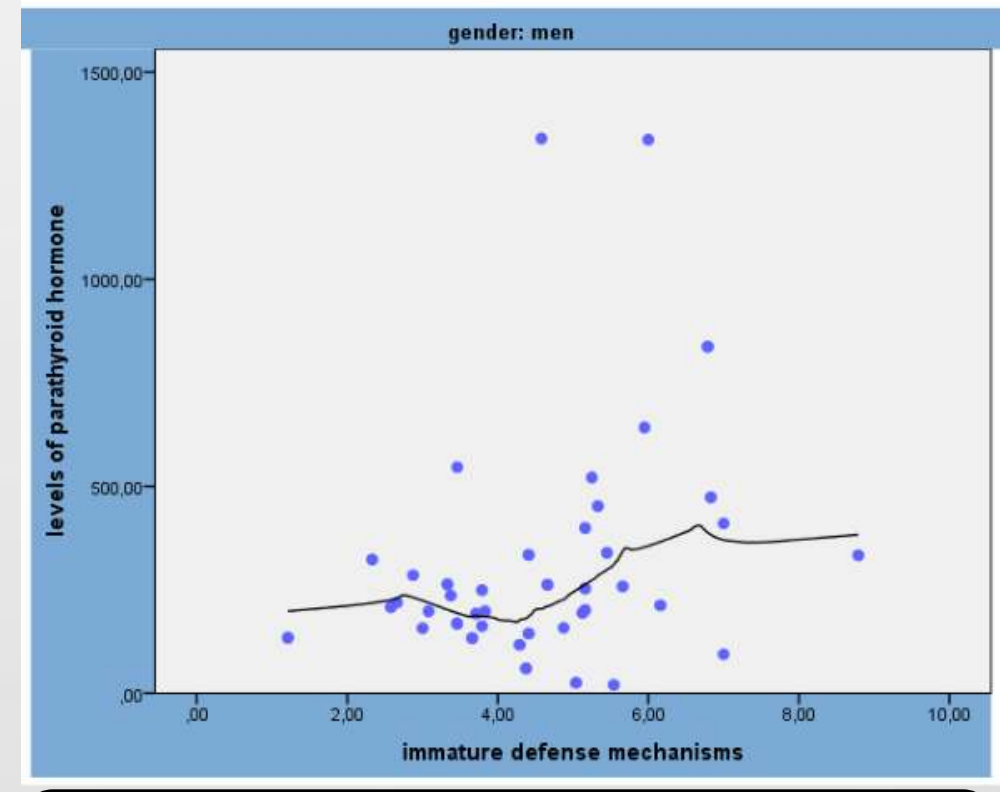

Immature defenses are in positive correlation with level of PTH in males (Spearman's rho $=0,317, \mathrm{p}=0,041$ ).

Conclusion:Patients on a haemodialysis use more mature and neurotic defensive mechanisms compared to immature defense mechanisms and this is first known survey on that topic among chronic haemodialysis patients. Levels of neurotic defense mechanisms are significantly higher in third shift compared to other two shifts. We found negative correlation between levels of mature and neurotic defense mechanisms and levels of parathyroid hormone in females and positive correlation between immature defense mechanisms and levels of parathyroid hormone in males. Connection between psychological and somatic is very complex and challenging and we wanted to point out that biopsychosocial approach should be used in everyday clinical practice.

\section{References:}

Costa RM, Brody S. Immature psychological defense mechanisms are associated with greater personal importance of junk food , alcohol, and television. Psychiatry Res. 2013;209(3):535-9.

2. Hyphantis TN, Triantafillidis JK, Pappa S, Mantas C, Kaltsouda A, Cherakakis P, Alamanos Y, Manousos ON MV. Defense mechanisms in inflammatory bowel disease. J Gastroenterol. 2005;40(1):24-30.

3. Poplašen D, Mar D, Ulovec Z, Brumen V. The use of assessment tools in a sample of Croatian professional fire-fighters. Psychiatr Danub. 2013;25(3):255-60. 